\title{
Comparative Analysis of Clinical Parameters and Sputum Biomarkers in Establishing the Relevance of Filamentous Fungi in Cystic Fibrosis
}

\section{OPEN ACCESS}

Edited by:

Jean-Philippe Bouchara,

Université d'Angers, France

Reviewed by:

Catherine Greene,

Royal College of Surgeons in Ireland,

Ireland

Geraint Rogers,

South Australian Health and Medical

Research Institute, Australia

*Correspondence:

Erol A. Gaillard

eag15@/eicester.ac.uk

Catherine H. Pashley

chp5@leicester.ac.uk

Specialty section:

This article was submitted to

Fungal Pathogenesis,

a section of the journal

Frontiers in Cellular and

Infection Microbiology

Received: 11 September 2020

Accepted: 04 December 2020

Published: 22 January 2021

Citation:

Patel D, Dacanay KC, Pashley $\mathrm{CH}$ and Gaillard EA (2021) Comparative Analysis of Clinical Parameters and

Sputum Biomarkers in Establishing the Relevance of Filamentous

Fungi in Cystic Fibrosis.

Front. Cell. Infect. Microbiol. 10:605241.

do: 10.3389/fcimb.2020.605241

\author{
Deepa Patel ${ }^{1,2}$, Keith Chester Dacanay ${ }^{1,3}$, Catherine H. Pashley ${ }^{1,3 *}$ \\ and Erol A. Gaillard ${ }^{1,2,3 *}$
}

\begin{abstract}
1 Department of Respiratory Sciences, University of Leicester, Leicester, United Kingdom, 2 Paediatric Respiratory Department, University Hospitals of Leicester NHS Trust, Leicester, United Kingdom, ${ }^{3}$ Institute for Lung Health, NIHR Respiratory Biomedical Research Center, Leicester, United Kingdom
\end{abstract}

Background: The relationship between fungal culture (FC) positivity and airway inflammation in CF is largely unknown. Identifying the clinical significance of filamentous fungi in CF using both clinical parameters and biomarkers may change our antimicrobial therapeutic strategies.

Objectives: To investigate the clinical characteristics and airway biomarker profile in relation to the detection of filamentous fungi in respiratory samples obtained from CF patients.

Methods: A prospective cohort study over 24 months, including children and adults with CF. Participants provided sputum and/or bronchoalveolar lavage samples, which underwent processing for bacterial and fungal culture, leukocyte differential cell count and biomarker analysis for neutrophil elastase (NE), interleukin-8 (IL-8), galactomannan and tumor necrosis factor receptor type 2 (TNF-R2). We performed FC using neat sputum plugs, an approach shown to be more sensitive compared to routine laboratory testing.

Results: Sixty-one patients provided 76 respiratory samples (72 sputum and 4 BAL). Median age was 17 years (range 6 months-59 years). FC positivity was noted in 49\% of the cohort. FC positivity was greater during pulmonary exacerbation compared to the stable state (67 versus 50\%). Participants aged 5-30 years had a lower FEV1 within the FC positive group. A significant association between FC positivity and non-tuberculosis mycobacterial (NTM) culture was observed on non-parametric testing ( $p=0.022)$ and regression analysis $(p=0.007)$. Exposure to indoor mold was a predictor for FC positivity $(p=0.047)$. There was a trend towards increased lung clearance index (LCl), bronchiectasis and intravenous antibiotic use in the FC positive group. There was no significant difference in biomarkers between FC positive and negative patients.

Conclusion: Aspergillus. fumigatus is the commonest filamentous fungi cultured from CF airways. We found no difference in the airway biomarker profile between FC positive and 
negative patients. The role of galactomannan and TNFR2 as fungal specific biomarkers in CF remains uncertain. $\mathrm{FC}$ positivity is associated with a lower $\mathrm{FEV}_{1}$ in younger patients, a lower $\mathrm{LCl}$, NTM positivity, bronchiectasis, and intravenous antibiotic exposure. Larger trials are needed to determine the role of galactomannan and TNF-R2 as potential fungal biomarkers in CF.

Keywords: biomarkers, inflammation, galactomannan, TNF-R2, IL-8, neutrophil, fungi, children

\section{INTRODUCTION}

Cystic fibrosis (CF) lung disease involves impaired mucociliary clearance leading to pulmonary inflammation, infection, and a more rapidly declining lung function. The CF airway milieu contains higher levels of pro-inflammatory cytokines in comparison to healthy controls (Sagel, 2009). Whilst the association between pathogenic bacteria and airway inflammation, infection and disease progression is well established (Hauser et al., 2011), less is known about the role of fungi in the CF airways and their impact on airway inflammation and lung disease progression. Allergic bronchopulmonary aspergillosis (ABPA) is a well-established complication in CF associated with sensitization to Aspergillus fumigatus (Thia and Balfour Lynn, 2009). In the absence of ABPA, it is not clear whether the detection of fungi in the CF airway in asymptomatic patients reflects simple colonization (innocent bystander effect) or infection contributing to increased airway inflammation (Liu et al., 2013; Chotirmall and McElvaney, 2014).

A. fumigatus is the commonest filamentous fungus isolated from CF airways (Pihet et al., 2009; Sudfeld et al., 2009). Improved mycological methods enable the detection of other fungi, including Aspergillus terreus, Scedosporium apiospermum complex, Exophiala dermatitidis, and Penicillium species (Borman et al., 2010).

The clinical significance of $A$. fumigatus colonization in CF is uncertain. Whilst Saunders et al. (2016) report a steeper decline in Forced Expiratory Volume in 1 second (FEV1) in their fungal culture positive pediatric CF cohort, studies by Baxter et al. (2013b) and De Vrankrijker et al. (2011) found no association between A. fumigatus airway colonization and lung function. Other studies have reported greater risk of pulmonary exacerbations requiring hospitalization (Amin, 2010) and higher rates of bronchiectasis (McMahon et al., 2012).

The lack of standardization in processing respiratory samples for fungal culture, differing fungal recovery rates between standard culture methods and the insensitivity of certain fungal culture methods (Chen et al., 2017) is likely to contribute to these contradictory findings. The use of modified culture methods using higher amounts of sputum inoculum have shown increased sensitivity in the detection of fungi (Pashley et al., 2012; Fraczek et al., 2014).

Exploring the relationship between FC positivity and inflammatory airway biomarkers may help in clarifying the clinical significance of fungi. Attempts at profiling the inflammatory cytokine profile in conjunction with clinical and microbiological profiles in CF is limited to a few well- established biomarkers (Frayman et al., 2017) such as neutrophil elastase (NE) and interleukin-8 (IL-8) found in higher concentrations in the CF airway (Frayman et al., 2017). There is a need to identify biomarkers that are specific for pulmonary infections including fungal infection. There is emerging evidence for the novel biomarkers galactomannan and tumor necrosis factor receptor 2 (TNF-R2) for monitoring non-invasive fungal lung disease in CF and asthma (Baxter et al., 2013a; Ghebre et al., 2017)

Neutrophil-driven airway inflammatory responses play a central role in CF. Activation of the innate immune system following pathogenic exposure results in phagocytosis, release of NE and cytokines (Hartl et al., 2012; Lammertyn et al., 2017). IL$1 \beta$ and TNF- $\alpha$ facilitate recruitment of immune cells to the site of inflammation. Recruitment cytokines such as IL-8 have high neutrophil attracting capacity (Strieter, 2002), further exacerbating the inflammatory process and resulting in large amounts of neutrophilic intracellular protease and oxidant being released. Activation cytokines (interferon- $\gamma$, IL-2, IL-12, and TNF- $\alpha$ ) augments the T-helper cell response.

Inhalation of fungal spores may result in germination and hyphal growth within the bronchioles (Chotirmall and McElvaney, 2014). Fungi may subsequently colonize, infect, or induce a spectrum of hypersensitivity reactions within the airways. Galactomannan, a fungal cell wall component, is released during hyphal tissue invasion by Aspergillus species and can be detected in body fluids (Mennink-Kersten et al., 2004). Current literature focusses on the role of galactomannan in invasive pulmonary aspergillosis, but only a few studies to date have aimed to evaluate its role in non-invasive fungal lung disease (Nguyen et al., 2007; Baxter et al., 2013a).

Differentiating between filamentous fungi as innocent bystanders and fungal infection, using a combination of clinical parameters and airway biomarkers, may inform antimicrobial strategies. This is particularly important given emerging evidence that CF transmembrane conductance regulator (CFTR) modulators may not alter susceptibility to $A$. fumigatus (Fritsch et al., 2019). Consequently the role of fungi in $\mathrm{CF}$ is likely to present an ongoing clinical challenge, whilst at the same time limiting our therapeutic options due to complex drug interactions (Tracy and Moss, 2018).

We report a prospective observational cohort study. Our principal aim was to describe the clinical characteristics and airway inflammatory biomarker profile (NE, IL-8, TNF-R2 and galactomannan) in filamentous fungi culture-positive CF patients. We hypothesized that fungal culture positivity in CF is associated with worse lung function and increased airway inflammation. 


\section{MATERIALS AND METHODS}

\section{Setting and Study Population}

Children and adults attending tertiary CF centers at the University Hospitals of Leicester NHS trust, UK, were eligible for enrolment if they had a well-documented diagnosis of CF based on the presence of two disease-causing CFTR mutations and/or a sweat chloride $\geq 60 \mathrm{mmol} / \mathrm{L}$. Exclusion criteria included CF transplant patients. Participants were approached during routine clinical encounters inclusive of inpatient admissions and outpatient visits. Inclusion criteria for this study included having sputum and/or bronchoalveolar (BAL) samples collected. All respiratory samples were obtained when clinically indicated. Samples during the stable state are obtained at all routine clinic visits on a two-monthly basis or as part of their annual comprehensive review process. Further respiratory samples were obtained during periods of exacerbation as defined by Fuch's criteria (Fuchs et al., 1994). BAL sampling was undertaken when there was no clinical response to a 4-6-week course of broad spectrum oral antibiotics without a causative pathogen on respiratory samples.

Written informed consent was obtained from study participants $\geq 17$ years of age. For participants $<17$ years of age written informed consent was obtained from parents or caregivers. Pediatric patients older than 7 years old also provided written assent. This study was approved for review and anonymized use of patient clinical data as part of the Leicester longitudinal study of respiratory infections and microbiomics in CF by the East Midlands Research Ethics Committee, reference number 12/WM/0285.

\section{Study Design}

Prospective cohort study, including pediatric and adult CF patients, from September 2016 to August 2018. The study protocol included a baseline visit which occurred at a routine clinical encounter during a non-exacerbation period. We obtained paired exacerbation and stable respiratory samples for all study participants who experienced a pulmonary exacerbation during the study period.

\section{Sputum and Data Collection}

Pediatric study participants aged five years and over underwent sputum induction (Paggiaro et al., 2002; Ahmed et al., 2019) lung function and serological testing as part of their annual review process during a period of stable disease. Sputum was induced with hypertonic saline in our purpose built negative pressure room dedicated to children. If an insufficient sputum sample was obtained during sputum induction, spontaneously expectorated sputum samples and/or BAL samples were collected according to standardized procedures (Miller et al., 2005). BAL samples were obtained in theatre under anesthesia as recommended by the ERS task force (De Blic et al., 2000). Adult patients do not routinely undergo sputum induction and therefore spontaneously expectorated sputum was used during a stable clinical state.

Electronic medical records were used to obtain study data including: patient demographics, CFTR genotype, medications, lung function, serological, clinical microbiology, and radiology results (bronchiectasis on CT chest). Pediatric patients also underwent a multiple nitrogen washout test (Lum et al., 2007; Davies et al., 2008; Nuttall et al., 2019) as part of their annual review process. We assessed the respiratory status (stable versus exacerbation) of all CF patients at the time of sputum collection.

\section{Respiratory Sample Processing and Storage}

For the detection of filamentous fungi, a sensitive approach was used whereby neat sputum plugs were inoculated onto potato dextrose agar plates containing $16 \mu \mathrm{l} / \mathrm{ml}$ chloramphenicol, $4 \mu \mathrm{l} / \mathrm{ml}$ gentamicin, and $5 \mu \mathrm{l} / \mathrm{ml}$ fluconazole (PGCF) (Pashley et al., 2012). Samples were incubated at $37^{\circ} \mathrm{C}$ for up to seven days. We identified fungi based on macroscopic and microscopic features (Greer et al., 1997). For bacterial cultures the British National Standard method for investigating sputum was used in accordance with the UK CF Trust Microbiology Standards Working Group recommendations (Public Health England, 2019). Sputum was homogenised with $0.1 \%$ dithiothreitol (DTT) and $10 \mu \mathrm{l}$ of this mixture was diluted in $5 \mathrm{ml}$ distilled water. A sterile loop was used to simultaneously inoculate $1 \mu \mathrm{l}$ onto the following culture media: (1) mannitol salt/chromogenic agar, (2) cystine-lactose-electrolyte-deficient (CLED) agar, (3) Burkholderia cepacia selective agar, and (4) Mycobacterium species selective agar at $35-37^{\circ} \mathrm{C}$. All samples were incubated for $48 \mathrm{~h}$ to five days, except for Mycobacterium selective culture, which requires incubation for up to eight weeks.

Supernatant from sputum and/or BAL samples were obtained following homogenisation with $0.1 \%$ DTT, followed by vortexing, filtering and a centrifugation step as per our local SOP (Bhatt, 2016).

\section{Cohort Characterization}

Participants were categorized into the following groups; A) fungal culture positive and B) fungal culture negative based on absence of filamentous fungi on culture. Further sub-group analysis included paired samples obtained during pulmonary exacerbation and a clinically stable state. Pulmonary exacerbation was defined by Fuch's criteria and stable disease included the absence of Fuch's criteria (Fuchs et al., 1994). Colonization with a known CF pathogen was defined as $\geq 50 \%$ respiratory samples positive for the same bacteria on routine culture within a 12-month period (Liu et al., 2013).

Participants were deemed to have allergic bronchopulmonary aspergillosis (ABPA) (Barton et al., 2008) if they had suggestive clinical features including; a total serum $\operatorname{IgE}>500 \mathrm{kU} / \mathrm{L}$ and a raised specific $\operatorname{IgE}$ to $A$. fumigatus of $>0.35 \mathrm{kUA} / \mathrm{L}$ (Thia and Balfour Lynn, 2009).

\section{Sputum Cell Differentials}

Cytoslides were prepared by fix air-drying in 100\% methanol for five minutes followed by staining using RapiDiff II (Bios Europe) staining kit according to protocol (Parker, 2018). The cytoslides were used to determine the leukocyte differential cell count (Brightling et al., 2000). 


\section{ELISA Biomarker Assays}

Inflammatory cytokine profiles were measured in sputum supernatants; We estimated NE using the PMN Human Elastase ELISA kit (Invitrogen ${ }^{\mathrm{TM}}$ ), IL-8 using the Human IL-8 ELISA Kit (ThermoFisher Scientific), galactomannan using the Platelia ${ }^{\mathrm{TM}}$ Enzyme Immunoassay Aspergillus antigen ${ }^{\odot}$ (Bio-Rad) kit and TNF-R2 using the Human Tumor Necrosis Factor Receptor II (soluble TNF-R2) ELISA kit (Invitrogen ${ }^{\mathrm{TM}}$ ). All ELISA assays were performed in accordance with individual manufacturer's instructions. Results of NE, IL-8, and TNF-R2 assays were interpreted using standard curves appropriate for individual kit protocols. Galactomannan was measured using ratios of optical densities (OD). A positive result was based on a GM optical density index (ODI) of $\geq 0.5$.

An optimization step was undertaken due to homogenization of our respiratory samples with DTT. All inflammatory mediator kits underwent preparation of standards with and without DTT for comparison. This validation step did not demonstrate any significant variations in the concentration of standards for biomarker assay kits.

\section{Statistical Analysis}

We present summary statistics as median (range) for continuous data and as frequency (\%) for categorical data. The comparison of baseline characteristics across both groups of fungal culture was performed using Mann-Whitney $U$ test for continuous data and Chi-squared tests for categorical data. Analysis of normally distributed data including the association of lung function with age was performed using the independent samples t-test. Statistical significance was at the 0.05 level. The association between clinical characteristics across both categories of fungal culture was investigated with the use of a binomial regression model and reported as p-values (Wald test) and odds ratios (OR). The following independent variables; age, antibiotic use, bronchiectasis, lung function and immunological markers on serology were used for this logistic regression model to determine the association with fungal culture positivity (dependent variable).

\section{RESULTS}

\section{Cohort Characteristics}

Sixty-one of the 73 patients enrolled provided respiratory specimens and were subsequently eligible for inclusion in our study. Twelve patients were excluded as no respiratory sample for fungal culture was obtained (Figure 1). A total of 76 respiratory specimens were processed for fungal culture, which comprised of 72 sputum and four BAL samples. Median age at recruitment was 17 years (range 6 months-59 years) with 29 male participants and a median percent predicted $\mathrm{FEV}_{1}$ of $77 \%$ (range 21-120\%). All 61 participants provided a sputum sample during the stable state, of whom 43 patients also produced respiratory samples suitable for biomarker analysis. Of the respiratory samples that underwent biomarker analysis $(\mathrm{n}=$ 43), only three samples were derived from BAL. Fifteen

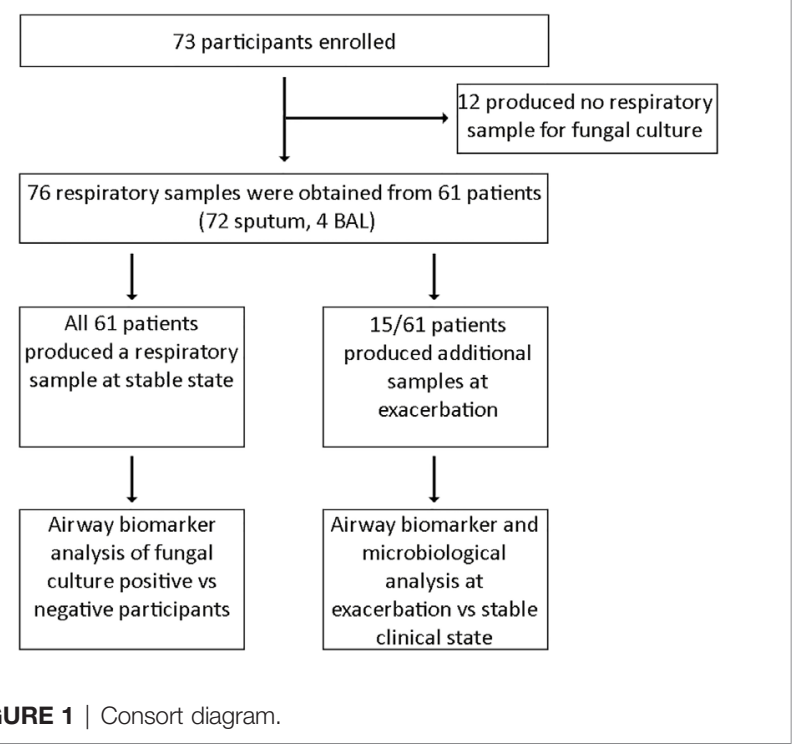

patients provided a further respiratory sample during clinical exacerbation to enable comparison with their paired stable state sample.

\section{Clinical Characteristics in Relation to Fungal Culture}

Thirty-one patients (49\%) cultured filamentous fungi from their sputum and/or BAL during a clinically stable state. Concomitant growth of A. fumigatus and yeast was the commonest $(60 \%)$ within the fungal culture positive group, followed by $A$. fumigatus alone (40\%). No other filamentous fungi were detected.

The median age within the fungal culture positive cohort was 15 years (range 7-53 years). No child under age seven years was observed to be fungal culture positive. Patient demographics, clinical data and cultured pathogens are presented in Table 1. No significant differences were observed between the distribution of age, BMI, genetics, indoor mold exposure (as defined by the presence of self-reported household mold) and pancreatic status between the two groups.

We observed significantly higher rates of non-tuberculous mycobacteria (NTM) isolates within the fungal culture positive sub-group ( $\mathrm{p}=0.022$ ). This finding was not noted for cocolonization with other bacteria such as Staphylococcus aureus or Pseudomonas aeruginosa.

We report greater use of inhaled corticosteroids within the FC positive cohort compared to the FC negative cohort ( $\mathrm{p}=0.008)$.

A binomial regression analysis was carried out to predict whether fungal culture positivity is associated with clinical characteristics including age, sex, mold exposure, routine microbiology, medication use, and lung function. Fungal culture positivity was 14 times more likely in the presence of NTM $(p=0.007)$ and five times more likely with indoor mold exposure $(\mathrm{p}=0.047)$. Although not statistically significant, the use of three-monthly intravenous antibiotic was four times more 
likely to be associated with fungal culture positivity. Significant associations for the predictor of fungal culture positivity were not demonstrated for other independent variables highlighted in Table 2. Missing values within the binomial regression model were accounted for by use of median values $(\mathrm{N}<4$ for independent variables including prophylactic, nebulized and intravenous antibiotic use, lung function, microbiology and serological markers). Due to multiple missing values for the independent variables LCI and bronchiectasis, a regression model could not be utilized without introducing significant bias.

The trend in lung function measures in relation to age and fungal culture positivity is shown in Figure 2. The mean FEV1 is lower within the fungal culture positive cohort at all ages with the exception of the over 30-year group. A significantly lower FEV1 was noted within the 21-30-year old fungal-culture positive group $(\mathrm{p}=$ 0.015). Although not statistically significant, we noted a higher FEV1 within the over 30-year old fungal-culture positive subgroup.

Although not significant, we also note an increased LCI within the fungal culture positive cohort at all ages in comparison to the fungal culture negative cohort.

Similar rates of ABPA were noted between the two cohorts with $13 \%$ in the fungal culture positive group $(n=4)$ and $19 \%$ in the fungal culture negative group $(n=6)$.

\section{Biomarker Analysis Neutrophil Elastase}

All samples were processed for NE however only 33/43 (77\%) yielded a result as ten readings were unavailable due to insufficient sample. Twenty four of the 33 samples (73\%) had a detectable $\mathrm{NE}$ value of $\geq 0.1 \mathrm{ng} / \mathrm{ml}$ (reference range $0.16-10 \mathrm{ng}$ / $\mathrm{ml}$ ). The remaining samples had a value of $<0.1 \mathrm{ng} / \mathrm{ml}$, which fell outside of the detectable range on the standard curve. There was no significant difference in NE between the fungal culture positive group (median value $0.1 \mathrm{ng} / \mathrm{ml}$, range $0.1-10$ ) and the fungal culture negative group (median $0.1 \mathrm{ng} / \mathrm{ml}$, range $0.1-2.3$, $\mathrm{p}=0.107$ ) (Figure 3A).

Two patients had high NE levels (> $10 \mathrm{ng} / \mathrm{ml}$ ), both of whom were fungal culture positive. The first patient had serological evidence of ABPA with a serum IgE of $4433 \mathrm{kU} / \mathrm{L}$, Aspergillus specific IgE of $25.1 \mathrm{kUA} / \mathrm{L}$, and an Aspergillus specific IgG of 130 $\mathrm{mg} / \mathrm{L}$. This patient was receiving three-monthly intravenous antibiotics and nebulized antibiotics as part of Mycobacterium abscessus eradication regime. The second patient had mixed growth of A. fumigatus and Haemophilus influenzae on sputum culture, no pre-existing history of fungal lung disease, normal serological markers, and was not receiving regular intravenous antibiotics.

TABLE 1 | Clinical characteristics in CF with and without positive sputum for filamentous fungi at stable state.

\begin{tabular}{|c|c|c|c|c|c|}
\hline \multirow[t]{2}{*}{ Variable } & \multicolumn{2}{|c|}{ Fungal culture positive } & \multicolumn{2}{|c|}{ Fungal culture negative } & \multirow[t]{2}{*}{$\mathbf{P}$ value } \\
\hline & Total N & & Total $\mathrm{N}$ & & \\
\hline Male n (\%) & 30 & $13(43 \%)$ & 31 & $16(52 \%)$ & 0.517 \\
\hline Age (years) & 30 & $15(7-53)$ & 31 & $16(0.5-59)$ & 0.919 \\
\hline DF508 homozygous n (\%) & 30 & $13(43 \%)$ & 31 & $14(45 \%)$ & 0.886 \\
\hline Pancreatic insufficient $\mathrm{n}(\%)$ & 30 & $26(87 \%)$ & 31 & $28(90 \%)$ & 0.654 \\
\hline $\mathrm{BMl}\left(\mathrm{kg} / \mathrm{m}^{2}\right)$ & 30 & $20.8(13.5-30.1)$ & 31 & $19.5(15.3-27.6)$ & 0.573 \\
\hline Indoor mold exposure n (\%) & 29 & $12(40 \%)$ & 30 & $9(29 \%)$ & 0.659 \\
\hline Ivacaftor n (\%) & 30 & $1(3 \%)$ & 31 & $3(10 \%)$ & - \\
\hline Prophylactic antibiotics n (\%) & 29 & $16(55 \%)$ & 30 & $17(56 \%)$ & 0.993 \\
\hline Nebulized antibiotics n (\%) & 29 & $14(48 \%)$ & 30 & $14(47 \%)$ & 0.992 \\
\hline Inhaled steroids n (\%) & 28 & 19 (68\%) & 31 & $10(32 \%)$ & 0.008 \\
\hline Oral steroids n (\%) & 29 & $3(10 \%)$ & 31 & $1(3 \%)$ & - \\
\hline 3 monthly ivab n (\%) & 29 & $11(38 \%)$ & 30 & $5(17 \%)$ & 0.185 \\
\hline SA colonization n (\%) & 29 & $6(21 \%)$ & 30 & $4(13 \%)$ & 0.753 \\
\hline PA colonization n (\%) & 29 & $8(28 \%)$ & 30 & $9(30 \%)$ & 0.979 \\
\hline Previous NTM n (\%) & 29 & $12(41 \%)$ & 30 & $3(10 \%)$ & 0.022 \\
\hline Bronchiectasis on CT $\mathrm{n}(\%)$ & 17 & $13(76 \%)$ & 11 & $7(64 \%)$ & 0.225 \\
\hline ABPA $n(\%)$ & 29 & $4(14 \%)$ & 30 & $6(20 \%)$ & - \\
\hline Total IgE (KU/L) & 29 & $156(5-4,433)$ & 29 & $76(3-4,273)$ & 0.260 \\
\hline Asp. IgE (KUA/L) & 27 & $0.33(0-41)$ & 30 & $0.05(0-37.8)$ & 0.378 \\
\hline Asp. IgG (mg/L) & 29 & $58(15-185)$ & 28 & $52(6.8-181)$ & 0.139 \\
\hline $\mathrm{FEV}_{1}$ predicted (\%) & 30 & $72(21-122)$ & 28 & $80(24-131)$ & 0.920 \\
\hline $\mathrm{FEV}_{1} / \mathrm{FVC}(\%)$ & 30 & $70(32-97)$ & 28 & $72(41-92)$ & 0.767 \\
\hline $\mathrm{LCl}$ & 9 & $11.6(7.75-20.72)$ & 11 & $7.84(6.41-13.85)$ & 0.972 \\
\hline Sputum lymphocyte count (\%) & 13 & $0(0-0.5)$ & 11 & $0(0-5.75)$ & 0.289 \\
\hline Sputum macrophage count (\%) & 13 & $2.75(0-32)$ & 11 & $2(0.25-28.25)$ & 0.511 \\
\hline Sputum eosinophil count (\%) & 13 & $0.1(0-2.75)$ & 11 & $0.25(0-14.5)$ & 0.631 \\
\hline Sputum neutrophil count (\%) & 13 & 94.88 (67.75-99.5) & 11 & $92.75(10.25-98.5)$ & 0.887 \\
\hline
\end{tabular}

IVAB, Intravenous antibiotics; ICS, inhaled corticosteroid; SA, Staphylococcal aureus; PA, Pseudomonas aeruginosa, NTM, non-tuberculous mycobacteria; IgE, Immunoglobulin E; Asp IgE, Aspergillus specific IgE; Asp IgG, Aspergillus specific immunoglobulin G; FEV1, Forced expiratory volume in one second; FVC, Forced vital capacity; LCl, Lung clearance index. Colonization is defined as $>50 \%$ samples positive over 12 -month period.

Categorical data is presented as frequency (n) and analyzed using Chi-squared test. Continuous data is presented as median and range. Non-parametric data was analyzed as comparison of medians across groups using the Mann-Whitney $U$ test. Statistical significance was defined as $P$ value $<0.05$.

Bold values signify statistical significance at $P<0.05$. 
TABLE 2 | Binomial regression analysis of clinical characteristics in relation to fungal culture positivity.

\begin{tabular}{lcc}
\hline Variable & P value & Exp(B)/OR 95\% Cl \\
\hline Age (years) & 0.542 & $1.024(0.948-1.107)$ \\
Sex & 0.220 & $0.354(0.067-1.860)$ \\
Pancreatic insufficiency & 0.469 & $0.167(0.396-7.486)$ \\
Indoor mold exposure & $\mathbf{0 . 0 4 7}$ & $5.217(1.025-26.545)$ \\
Prophylactic antibiotic use & 0.546 & $0.635(0.146-2.773)$ \\
Nebulized antibiotic use & 0.801 & $0.822(0.180-3.750)$ \\
3 monthly ivab exposure & 0.108 & $4.761(0.712-31.836)$ \\
FEV 1 predicted (\%) & 0.351 & $0.985(0.953-1.017)$ \\
S. aureus & 0.514 & $1.845(0.293-11.601)$ \\
P. aeruginosa & 0.869 & $1.175(0.170-7.984)$ \\
NTM & $\mathbf{0 . 0 0 7}$ & $14.263(2.064-98.540)$ \\
Total lgE (KU/L) & 0.264 & $1.001(0.999-1.002)$ \\
Asp. IgE (KUA/L) & 0.307 & $0.930(0.810-1.069)$ \\
Asp. IgG (mg/L) & 0.401 & $1.007(0.990-1.025)$
\end{tabular}

IVAB, Intravenous antibiotics; Forced expiratory volume in one second; IgE, Immunoglobulin E; Asp IgE, Aspergillus specific IgE; Asp IgG, Aspergillus specific immunoglobulin $G$. $P$ values are derived from the Wald test with statistical significance defined as $<0.05$.

\section{Interleukin-8}

Sufficient sputum was available for 23 samples to be tested for IL8 (reference range $15.6-1,000 \mathrm{pg} / \mathrm{ml}$ ). All assays yielded a detectable result of $>62 \mathrm{pg} / \mathrm{ml}$ (reference range 15.6-1,000 pg/ $\mathrm{ml}$ ) (Figure 3B). Sixteen of the 23 respiratory samples (70\%) tested were from fungal culture positive patients. The comparative median and ranges of IL- 8 between the fungal culture positive and negative groups are reported in Figure 3. There was no significant difference in IL- 8 between the fungal culture positive and negative sub-groups $(\mathrm{p}=0.871)$.

\section{Tumor Necrosis Factor Receptor 2}

All respiratory samples were tested for TNF-R2 (reference range 2-142 $\mathrm{ng} / \mathrm{ml}$ ). Eight patients (19\%) had a detectable level of $\geq 2$

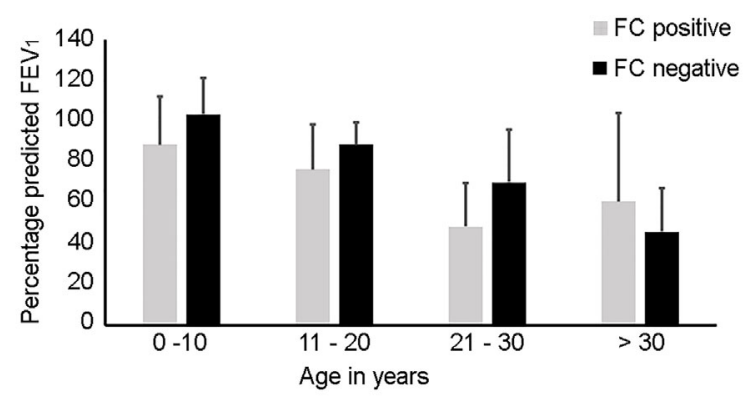

FIGURE 2 | Histogram representing differing age groups in relation to fungal culture (FC) positivity and percentage predicted Forced Expiratory Volume in 1 second $\left(\mathrm{FEV}_{1}\right)$. The lung function data is presented as the standard error of mean per age category. The mean $\mathrm{FEV}_{1}$ is lower within all age groups within the FC positive group with the exception of the over 30-year sub-group; 0-10 years (FC positive mean 89\%, 95\% Cl 64-113; FC negative mean 103\%, $95 \% \mathrm{Cl} 84-122, \mathrm{p}=0.25), 11-20$ years ( $\mathrm{FC}$ positive mean $76 \%, 95 \% \mathrm{Cl} 63-$ 90; FC negative mean $89 \%, 95 \% \mathrm{Cl} 77-100, \mathrm{p}=0.225$ ), $21-30$ years (FC positive mean 48\%, 95\% Cl 29-68; FC negative mean 70\%, 95\% Cl 48-92, $\mathrm{p}=0.015$ ) and $>30$ years (FC positive mean $61 \%, 95 \% \mathrm{Cl} 8-129$; FC negative mean 46\%, Cl 28-64, $p=0.434$ ). ng/ml (Figure 3C). Five of the eight were fungal culture positive. Both groups had a median TNF-R2 below the detectable range of the assay.

Participants with detectable TNF-R2 levels were not noted to have any discernible patterns to lung function impairment, lung clearance index, serological markers, intravenous antibiotic use, or radiological evidence of bronchiectasis on CT.

\section{Galactomannan}

There was enough sputum for galactomannan testing available for 32 patients. Only four had a positive result with $\geq 0.5$ ODI, three of which fell into the fungal culture positive group (Figure 3B).

\section{Paired Sputum Analysis}

Fifteen patients provided paired sputum samples obtained at routine exacerbation and stable state visits. Ten (67\%) were positive for filamentous fungi during an exacerbation state and seven (50\%) during the stable state. All but two participants' samples were culture positive for A. fumigatus. The other two samples were positive for either Penicillium species or Aspergillus niger, both during the acute exacerbation state. Figure 4 demonstrates a higher proportion of concomitant bacterial growth during the exacerbation state. Ninety-three percent had mixed bacterial and fungal growth during acute exacerbation in comparison to $60 \%$ during the stable state. Seven patients had the same bacterial species detected during exacerbation and stable states and represented chronic colonization. No participants during exacerbation were fungal culture negative and exhibiting only normal respiratory flora, compared to $20 \%$ $(\mathrm{n}=3)$ at stable state.

Due to a lack of sufficient sputum volume, only three paired samples were available for biomarker comparison between pulmonary exacerbation and stable disease. NE $(1.2 \mathrm{ng} / \mathrm{ml}$ exacerbation vs $0.05 \mathrm{ng} / \mathrm{ml}$ during stable state) and GM (0.5 OD exacerbation vs 0.14 OD stable) were higher during pulmonary exacerbation $(\mathrm{p}<0.001)$, with a trend towards higher IL-8 levels during the exacerbation state $(1,842.4 \mathrm{pg} / \mathrm{ml}$ exacerbation vs $1,335.3 \mathrm{pg} / \mathrm{ml}$ during stable).

\section{DISCUSSION}

Our study is the first to describe CF airway biomarkers in relation to fungal culture positivity. We report the relationship between airway fungal culture positivity, airway biomarkers and clinical, radiological and microbiological characteristics in children and adults with CF.

The clinical relevance of fungal airway colonization and its impact on lung disease progression in CF is unclear. In keeping with previous reports, we found that $A$. fumigatus was the most frequently isolated fungal species (Pihet et al., 2009). Colonization with $A$. fumigatus has been associated with more advanced CF lung disease (Ramsey et al., 2014), lower lung function (Amin, 2010; Saunders et al., 2016) and greater risk of pulmonary exacerbations requiring hospitalization 

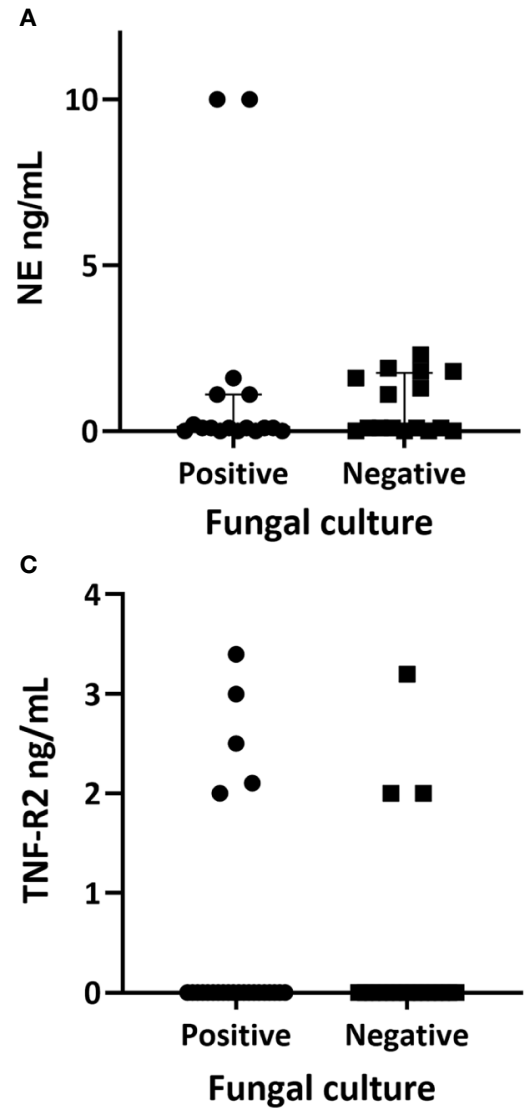
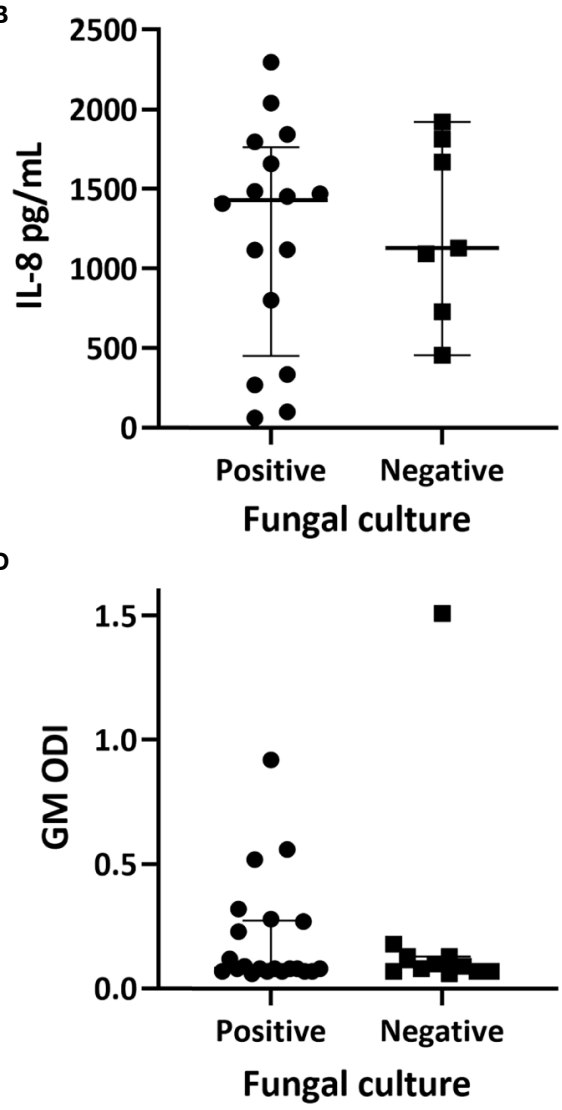

FIGURE 3 | Quantitative airway biomarker measurements in relation to fungal culture positivity. (A) neutrophil elastase with the fungal culture positive group having a median value of $0.1 \mathrm{ng} / \mathrm{ml}$ (range $0-10$ ) and the culture negative group had a median of 0.1 (range 0-2.3); $p=0.107$. (B) interleukin-8 with a median of 1,430.45 pg/ $\mathrm{ml}$ (range 62.9-2,296.2) within the fungal culture positive group and a median of 1,128.3 (range 455.2-1,921.7) within the fungal culture negative group; $p=0.871$. (C) tumour necrosis factor receptor 2 (TNF-R2) with a median of $<2 \mathrm{ng} / \mathrm{ml}$ within both subgroups. (D) galactomannan values represented as optical density index $(\mathrm{ODI})$ with the fungal culture group having a median of 0.09 (range $0.06-0.92$ ) and the culture negative group with a median of 0.08 (range $0.066-1.44$ ); $p=0.143$. Individual data, median and interquartile range are shown. Comparison of medians between groups were undertaken using the Mann-Whitney $U$ test for nonparametric data.

(Amin, 2010). In our study, the fungal culture positive group, with the exception of the over 30-year old sub-group, had a lower mean FEV1 across all younger age groups. Our findings of a higher FEV1 within the FC positive subgroup for the over 30year age group, may be a reflection of participants surviving with milder CF lung disease.

LCI derived from multiple breath washout is a more sensitive marker of lung disease progression compared to spirometry, allowing non-invasive monitoring of small airways disease. A study by Walicka-Serzysko et al. demonstrated a higher LCI in CF patients with A. fumigatus (Walicka-Serzysko et al., 2019). We also found higher LCI's in fungal culture positive patient but this did not reach statistical significance.

The primary focus of previous studies have included exploring the association between A. fumigatus and clinical outcomes, there is a paucity of information exploring fungal airway colonization and its impact on markers of airway inflammation. It is well established that the CF airway milieu contains higher levels of pro-inflammatory cytokines in comparison to healthy controls (Sagel, 2009). Evidence for pulmonary inflammation in surveillance BAL such as raised NE and IL-8 in the CF airways is present, even in the absence of clinically apparent lung disease (Armstrong et al., 2005; Frayman et al., 2017; Khan et al., 2019). Sly et al. have reported a link between high NE levels within the CF airways and the subsequent development of bronchiectasis (Sly et al., 2009). Whether fungal colonization exacerbates underlying airway inflammation, resulting in higher levels of airway biomarkers and therefore further driving lung disease progression requires further study. Exploring this relationship is complicated by the difficulty in separating the effect of bacteria and fungi present in respiratory cultures.

In our study, only one-third of patients were fungal culture positive without the presence of concomitant pathogenic 

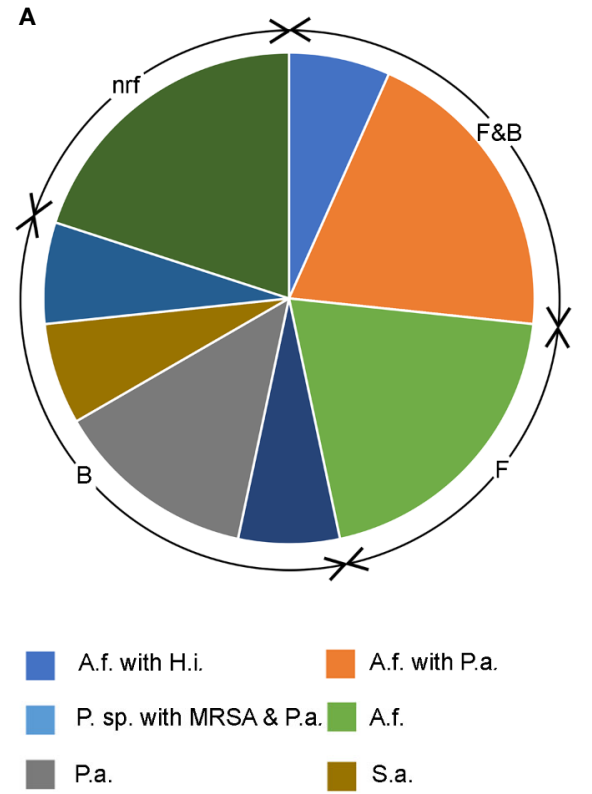
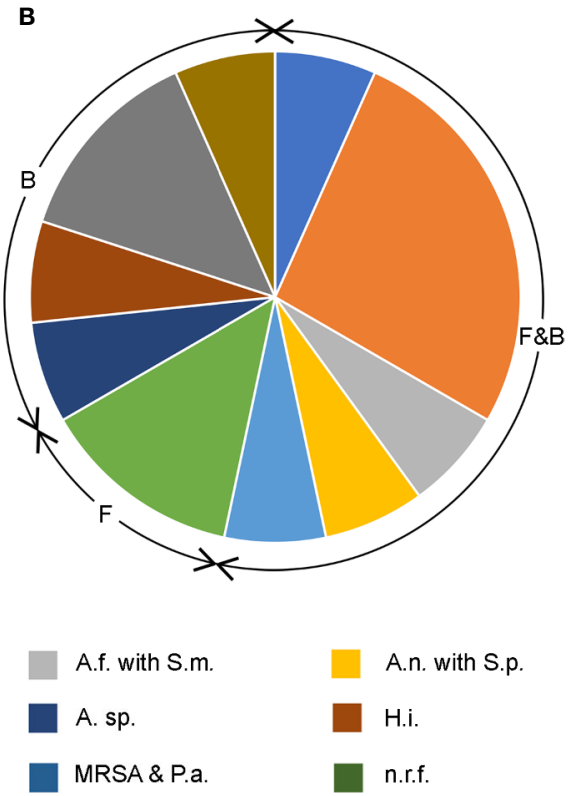

FIGURE 4 | Pie charts showing bacterial and filamentous fungal isolates cultured from respiratory samples during (A) stable state and (B) acute exacerbation. F\&B; concomitant fungal and bacterial growth, F; fungal only, B; bacterial only, nrf; normal respiratory flora. Fungi cultured: A.f. Aspergillus fumigatus, A.n. Aspergillus niger, P. sp. Penicillium sp. Bacteria cultured: A. sp. Achromobacter sp., H.i. Haemophilus influenzae, P.a. Pseudomonas aeruginosa, S.a. Staphylococcus aureus, MRSA methicillin resistant S. aureus, S.m. Strenotrophomonas maltophilia, S.p. Streptococcus pneumoniae.

bacteria, making the direct attribution of inflammation to a positive fungal culture problematic. We found no significant difference between NE and IL- 8 between the fungal culture positive and negative groups. Two patients with very high levels of NE were fungal culture positive but these low numbers preclude any firm conclusion. Studies have shown improvements in NE and IL-8 levels following treatment of an exacerbation (Ordonez et al., 2003; Colombo et al., 2005). Evidence suggests NE activity is higher during exacerbation and is responsive to antibiotics (Chalmers et al., 2017). Whilst we acknowledge our small number of paired patient samples due to difficulty in obtaining sufficient sputum volumes, we report higher levels of NE and IL-8 during pulmonary exacerbation compared to the stable state.

Galactomannan may be a useful screening test for invasive pulmonary aspergillosis (Kimura et al., 2009; Talento et al., 2017). The diagnostic usefulness of galactomannan assays in patients with non-invasive fungal lung disease in $\mathrm{CF}$, such as ABPA and chronic pulmonary aspergillosis remains uncertain (Nguyen et al., 2007; Kitasato et al., 2009; Fayemiwo et al., 2017). We found that only four patients (13\%) within our cohort had a positive result for galactomannan. There were no significant difference in the absolute values of galactomannan between the fungal culture positive and negative groups. Contrary to our study findings, galactomannan has been reported as a sensitive method of detecting Aspergillus in sputum in comparison to fungal cultures (Baxter et al., 2013a), even in the absence of invasive disease. Baxter et al. used sputum samples homogenized with Sputasol (commercial product containing DTT) and sonication. In comparison we used homogenized sputum supernatants without sonication, which may have decreased the overall yield.

There is emerging evidence implicating TNF-R2 as a possible airway biomarker in fungal associated asthma. Ghebre et al. noted significantly higher TNF-R2 levels in adults with asthma who were fungal culture positive (Ghebre et al., 2017). Our study is the first to analyze TNF-R2 as a potential biomarker of inflammation in adults and children with cystic fibrosis. Overall, we detected TNF-R2 in only $19 \%$ of our patients. Within the limitations of our modest number of patients with detectable TNF-R2 levels, we found no association with adverse clinical outcomes. Whilst Ghebre et al. concluded that TNF-R2 may diminish the inflammatory response to A. fumigatus, the exact mechanism for this and its clinical implications in CF is unknown. Interestingly, Ghebre et al. concluded that TNF-R2 appears to be a strong discriminator of exacerbation in children and adults with asthma (Ghebre et al., 2019). Further studies in CF are required to determine if a similar relationship exists between fungal culture positivity, exacerbation states and higher TNF-R2 levels.

We found that the fungal culture positive subgroup had greater previous and/or current positive sputum isolates for non-tuberculous mycobacteria. This is in keeping with previous literature where it has been proposed that Mycobacterium superinfection can occur alongside aspergillosis (Cui et al., 2013). The proposed theories include a synergistic relationship which enhances their resistance to environmental 
change such as use of broad-spectrum antibiotics (Kerr, 1994; Cui et al., 2013).

Within our CF cohort, we report higher rates of fungal culture positivity with indoor mold exposure. Evidence suggests a link between indoor fungal contamination and ABPA. Reports linking airway fungal colonization and mold exposure in CF are scarce (Rocchi et al., 2015). Fairs et al. reported environmental mold exposure may predispose asthmatics to airway colonization (Fairs et al., 2013) and whether this also occurs in CF patients is unclear and requires further study.

Although not statistically significant we observed a higher rate of bronchiectasis in the fungal culture positive cohort with $76 \%$ having radiological evidence of this on CT chest, in comparison to $64 \%$ of the culture negative cohort. Similar associations have been reported between A. fumigatus and higher radiological scores with more significant bronchiectasis (McMahon et al., 2012).

Whilst over 35\% of our fungal culture positive cohort received three-monthly intravenous antibiotics, only $16 \%$ of fungal culture negative group were exposed to regular antibiotics. Several studies have shown an association between the use of broad-spectrum antimicrobials and A. fumigatus airway colonization (Burns et al., 1998; Hodson et al., 2002). The use of regular intravenous antibiotics are typically reserved for patients with more severe lung disease and/or bacterial colonization. The concept of regular exposure to broad spectrum antimicrobials reducing competition for surfaces and limited nutrients, therefore promoting fungal adhesion, growth and colonization has therefore been proposed (Oever and Netea, 2014). However, a study by Baxter et al. reported a significant decline in the bioburden of Aspergillus when they compared preand post-antibiotic sputum samples from 26 adult CF patients (Baxter et al., 2013b). Therefore, the use of antimicrobials for well-established CF pathogens and its impact on A. fumigatus remains poorly understood.

We report a significantly higher rate of inhaled corticosteroid use within the FC positive cohort. This is a well-established finding in existing literature whereby the use of inhaled and/or systemic corticosteroids are known to promote A. fumigatus growth (Noni et al., 2015).

Our study limitations include a modest sample size, in particular for the paired sample analysis. The presence of significant numbers of non-sputum producing children in our study cohort means that we were unable to obtain sufficient volumes to complete ELISA biomarker and sputum cell differential analysis for all patients. We included a combination of sputum and BAL samples to enhance our sample size and to allow inclusion of non-sputum producing children within our study cohort. In order to minimize bias resulting from salivary contamination of respiratory samples, we included BAL samples when clinically indicated and where we were unable to obtain spontaneous or induced sputum samples.

Secondly, published studies report a wide range of prevalence in the detection of filamentous fungi (Nagano et al., 2010; Pashley et al., 2012) due to sampling techniques and culture conditions, it is likely that the true prevalence of fungi in CF is underestimated. To overcome this we used a modified fungal culture approach, which has been shown to be a more sensitive for the detection of filamentous fungi (Pashley et al., 2012). Whilst it is possible that our approach is detecting low levels of fungi that are not truly colonizing the lower airways, the association between higher culture rates and worse lung function in both this and the adult asthma studies (Fairs et al., 2010; Agbetile et al., 2012) that have utilized this more sensitive culture method would suggest the detection may be clinically relevant. An alternative approach to culture may be to utilize a molecular technique such as quantitative polymerase chain reaction (qPCR), which is commercially available for $A$. fumigatus and a limited number of other Aspergillus species (Guegan et al., 2018). Both modified fungal culture and Aspergillus quantitative PCR have been shown to be more sensitive than the NHS routine approach (Public Health England, 2019) for identifying A. fumigatus from respiratory secretions (Fraczek et al., 2014). It should be noted, however, that the current commercial qPCR assays, whilst based on quantitative technology, are used for determining the presence or absence of a fungus only, and will amplify DNA from dead as well as live fungal material.

Our study reports on the short-term clinical outcomes associated with FC positivity in CF given the cross-sectional methodology used. Whilst the long-term outcomes of ABPA and serological sensitization are well-described with evidence of lung function decline (Kraemer et al., 2006), increased need for intravenous antibiotics (Peetermans et al., 2014) and bronchiectasis (Agarwal et al., 2010), literature on the outcomes of Aspergillus colonization and non-invasive infection is scarce. It is clear that further prospective longitudinal studies are required to describe the clinical outcomes of A. fumigatus colonization and noninvasive infection.

Finally, statistical analysis for LCI and bronchiectasis were excluded from the binomial regression model due to the risk of bias from missing data. A limited number of patients had LCI results available as this is not routine practice in our adult CF cohort and is carried out as part of the annual comprehensive review process in children. However, not all children are capable of completing this procedure. We also note a limited number of patients underwent a CT chest as this was only carried out as part of routine care when indicated. In addition, our results obtained for NTM and mold exposure have high confidence intervals despite clinical significance, reflecting the highly variant estimates derived for these parameters.

In conclusion, we found no difference between the airway biomarker profile between sputum fungal culture positive and negative patients with $\mathrm{CF}$. The role of galactomannan and TNFR2 as more fungal specific airway biomarkers in CF remains uncertain. Fungal culture positivity is associated with a lower FEV1 in patients less than 30 years old, a lower LCI, higher rates of NTM positivity, bronchiectasis and intravenous antibiotic exposure. Larger trials using a broader range of inflammatory markers in conjunction with fungal culture and quantitative PCR measures are needed. 


\section{DATA AVAILABILITY STATEMENT}

The original contributions presented in the study are included in the article/supplementary materials. Further inquiries can be directed to the corresponding authors.

\section{ETHICS STATEMENT}

The studies involving human participants were reviewed and approved by Leicester longitudinal study of respiratory infections and microbiomics in CF by the East Midlands Research Ethics Committee, reference number 12/WM/0285. Written informed consent to participate in this study was provided by the participants' legal guardian/next of kin.

\section{AUTHOR CONTRIBUTIONS}

DP, CP, and EG co-designed the study. DP was responsible for analyzing the data and writing the manuscript. CP supervised the laboratory analyses (modified culture and ELISA) and EG the clinical aspects of the study. DP was responsible for patient

\section{REFERENCES}

Agarwal, R., Hazarika, B., Gupta, D., Aggarwal, A. N., Chakrabarti, A., and Jindal, S. K. (2010). Aspergillus hypersensitivity in patients with chronic obstructive pulmonary disease: COPD as a risk factor for ABPA? Med. Mycology 48 (7), 988-994. doi: 10.3109/13693781003743148

Agbetile, J., Fairs, A., Desai, D., Hargadon, B., Bourne, M., Mutalithas, K., et al. (2012). Isolation of filamentous fungi from sputum in asthma is associated with reduced post-bronchodilator FEV1. Clin. Exp. Allergy 42 (5), 782-791. doi: $10.1111 /$ j.1365-2222.2012.03987

Ahmed, M. I., Kulkarni, H., Shajpal, S., Patel, D., Patel, P., Claydon, A., et al. (2019). Early detection of non-tuberculous mycobacteria in children with cystic fibrosis using induced sputum at annual review. Pediatr. Pulmonol. 54 (3), 257-263. doi: 10.1002/ppul.24220

Amin, R. (2010). The effect of chronic infection with Aspergillus fumigatus on lung function and hospitalization in patients with cystic fibrosis. Chest 137 (1), 171-176. doi: 10.1378/chest.09-1103

Armstrong, D. S., Hook, S. M., Jamsen, K. M., Nixon, G. M., Carzino, R., Carlin, J. B., et al. (2005). Lower Airway Inflammation in Infants with Cystic Fibrosis Detected by Newborn Screening. Pediatr. Pulmonol. 40 (6), 500-510. doi: 10.1002/ppul.20294

Barton, R. C., Hobson, R. P., Denton, M., Peckham, D., Brownlee, K., Conway, S., et al. (2008). Serologic diagnosis of allergic bronchopulmonary aspergillosis in patients with cystic fibrosis through the detection of immunoglobulin $G$ to Aspergillus fumigatus. Diagn. Microbiol. Infect. Dis. 62 (3), 287-291. doi: 10.1016/j.diagmicrobio.2008.06.018

Baxter, C. G., Dunn, G., Jones, A. M., Webb, K., Gore, R., Richardson, M. D., et al. (2013a). Novel immunologic classification of aspergillosis in adult cystic fibrosis. J. Allergy Clin. Immunol. 132 (3), 560-566.e10. doi: 10.1016/ j.jaci.2013.04.007

Baxter, C. G., Moore, C. B., Jones, A. M., Webb, A. K., and Denning, D. W. (2013b). IgE-Mediated Immune Responses and Airway Detection of Aspergillus and Candida in Adult Cystic Fibrosis. Chest 143 (5), 1351-1357. doi: $10.1378 /$ chest.12-1363

Bhatt, H. (2016). SOP: Sputum processing procedure (Respiratory Biomedical Research Unit).

Borman, A. M., Palmer, M. D., Delhaes, L., Carrère, J., Favennec, L., Ranque, S., et al. (2010). Lack of standardization in the procedures for mycological recruitment, the design and custodianship of the clinical database, and processing clinical samples. DP and KD undertook the ELISA analysis. All authors contributed to the article and approved the submitted version.

\section{FUNDING}

Funding from Gilead Sciences Grant Program (grant no. 02261) and University Hospitals of Leicester charitable funds was awarded for this study alongside our separate study termed "Changes in the Cystic Fibrosis Airway Mycobiome Over Time and its Link with Bacterial Species Rarefication".

\section{ACKNOWLEDGMENTS}

$\mathrm{CP}$ and EG are supported by the NIHR Leicester Biomedical Research Centre and the Midlands Asthma and Allergy Research Association (MAARA). The views expressed are those of the authors and not necessarily those of the NHS the NIHR or the Department of Health.

examination of sputum samples from CF patients: a possible cause for variations in the prevalence of filamentous fungi. Med. Mycology 48 (O1), S88-S97. doi: 10.3109/13693786.2010.511287

Brightling, C. E., Monteiro, W., Ward, R., Parker, D., Morgan, M. D., Wardlaw, A. J., et al. (2000). Sputum eosinophilia and short-term response to prednisolone in chronic obstructive pulmonary disease: a randomised controlled trial. Lancet (British edition) 356 (9240), 1480-1485. doi: 10.1016/S0140-6736(00)02872-5

Burns, J. L., Emerson, J., Stapp, J. R., Yim, D. L., Krzewinski, J., Louden, L., et al. (1998). Microbiology of Sputum from Patients at Cystic Fibrosis Centers in the United States. Clin. Infect. Dis. 27 (1), 158-163. doi: 10.1086/514631

Chalmers, J. D., Moffitt, K. L., Suarez-Cuartin, G., Sibila, O., Finch, S., Furrie, E., et al. (2017). Neutrophil Elastase Activity Is Associated with Exacerbations and Lung Function Decline in Bronchiectasis. Am. J. Respir. Crit. Care Med. 195 (10), 1384-1393. doi: 10.1164/rccm.201605-1027OC

Chen, S. C., Meyer, W., and Pashley, C. H. (2017). Challenges in Laboratory Detection of Fungal Pathogens in the Airways of Cystic Fibrosis Patients. Mycopathologia (1975) 183 (1), 89-100. doi: 10.1007/s11046-017-0150-8

Chotirmall, S. H., and McElvaney, N. G. (2014). Fungi in the cystic fibrosis lung: Bystanders or pathogens? Int. J. Biochem. Cell Biol. pp, 161-173. doi: 10.1016/ j.biocel.2014.03.001

Colombo, C., Costantini, D., Rocchi, A., Cariani, L., Garlaschi, M. L., Tirelli, S., et al. (2005). Cytokine levels in sputum of cystic fibrosis patients before and after antibiotic therapy. Pediatr. Pulmonol. 40 (1), 15-21. doi: 10.1002/ ppul.20237

Cui, L., Morris, A., and Ghedin, E. (2013). The human mycobiome in health and disease. Genome Med. 5 (7), 63. doi: 10.1186/gm467

Davies, J. C., Cunningham, S., Alton, E. W.F.W., and Innes, J. A. (2008). Lung clearance index in CF: a sensitive marker of lung disease severity. Thorax 63 (2), 96-97. doi: 10.1136/thx.2007.077784

De Blic, J., Midulla, F., Barbato, A., Clement, D.III, Eber, E., Green, C., et al. (2000). Ers Task Force on bronchoalveolar lavage in children and Members of the Task Force: 'Bronchoalveolar lavage in children'. Eur. Respir. J. 15 (1), 217. doi: 10.1183/09031936.00.15121700

De Vrankrijker, A. M. M., van der Ent, C. K., van Berkhout, F. T., Stellato, R. K., Willems, R. J. L., Bonten, M. J. M., et al. (2011). 'Aspergillus fumigatus colonization in cystic fibrosis: implications for lung function?'. Clin. Microbiol. Infect. 17 (9), 1381-1386. doi: 10.1111/j.1469-0691.2010.03429.x 
Fairs, A., Agbetile, J., Hargadon, B., Bourne, M., Monteiro, W. R., Brightling, C. E., et al. (2010). IgE sensitization to aspergillus fumigatus is associated with reduced lung function in asthma. Am. J. Respir. Crit. Care Med. 182 (11), 1362-1368. doi: 10.1164/rccm.201001-0087oc

Fairs, A., Agbetile, J., Bourne, M., Hargadon, B., Monteiro, W. R., Morley, J. P., et al. (2013). Isolation of Aspergillus fumigatus from sputum is associated with elevated airborne levels in homes of patients with asthma. Indoor Air 23 (4), 275-284. doi: 10.1111/ina.12020

Fayemiwo, S., Moore, C. B., Foden, P., Denning, D. W., and Richardson, M. D. (2017). Comparative performance of Aspergillus galactomannan ELISA and PCR in sputum from patients with ABPA and CPA. J. Microbiol. Methods 140, 32-39. doi: 10.1016/j.mimet.2017.06.016

Fraczek, M. G., Kirwan, M. B., Moore, C. B., Morris, J., Denning, D. W., and Richardson, M. D. (2014). Volume dependency for culture of fungi from respiratory secretions and increased sensitivity of Aspergillus quantitative PCR. Mycoses 57 (2), 69-78. doi: 10.1111/myc.12103

Frayman, K. B., Armstrong, D. S., Carzino, R., Ferkol, T. W., Grimwood, K., Storch, G. A., et al. (2017). The lower airway microbiota in early cystic fibrosis lung disease: a longitudinal analysis. Thorax 72 (12), 1104. doi: 10.1136/ thoraxjnl-2016-209279

Fritsch, N., Green, H., Jones, A., and Barry, P. (2019). S16 The influence of the CFTR modulator ivacaftor on aspergillosis in cystic fibrosis. Thorax 74 (Suppl 2), A11. doi: 10.1136/thorax-2019-BTSabstracts2019.22

Fuchs, H. J., Borowitz, D. S., Christiansen, D. H., Morris, E. M., Nash, M. L., Ramsey, B. W., et al. (1994). Effect of aerosolized recombinant human DNase on exacerbations of respiratory symptoms and on pulmonary function in patients with cystic fibrosis. The Pulmozyme Study Group. New Engl. J. Med. 331 (10), 637. doi: 10.1056/NEJM199409083311003

Ghebre, M. A., Desai, D., Singapuri, A., Woods, J., Rapley, L., Cohen, S., et al. (2017). 'Sputum Inflammatory Mediators Are Increased in Aspergillus fumigatus Culture-Positive Asthmatics'. Allergy Asthma Immunol. Res. 9 (2), 177-181. doi: 10.4168/aair.2017.9.2.177

Ghebre, M. A., Pang, P. H., Desai, D., Hargadon, B., Newby, C., Woods, J., et al. (2019). 'Severe exacerbations in moderate-to-severe asthmatics are associated with increased pro-inflammatory and type 1 mediators in sputum and serum'. BMC Pulmonary Med. 19 (1), 144. doi: 10.1186/s12890-019-0906-7

Greer, D., Campbell, C. K., Johnson, E. M., Philpot, C. M., and Warnock, (1997). Identification of pathogenic fungi, Public Health Laboratory Service, London, (1996). J. Am. Acad. Dermatol. 36 (6), 1033. doi: 10.1016/S0190-9622(97)80307-6

Guegan, H., Chevrier, S., Belleguic, C., Deneuville, E., Robert-Gangneux, F., and Gangneux, J. (2018). Performance of molecular approaches for aspergillus detection and azole resistance surveillance in cystic fibrosis. Front. Microbiol. 9, 531. doi: $10.3389 /$ fmicb.2018.00531

Hartl, D., Gaggar, A., Bruscia, E., Hector, A., Marcos, V., Jung, A., et al. (2012). Innate immunity in cystic fibrosis lung disease. J. Cystic Fibrosis 11 (5), 363382. doi: 10.1016/j.jcf.2012.07.003

Hauser, A. R., Jain, M., Bar-Meir, M., and McColley, S. A. (2011). Clinical Significance of Microbial Infection and Adaptation in Cystic Fibrosis. Clin. Microbiol. Rev. 24pp (1), 29-70. doi: 10.1128/CMR.00036-10

Hodson, M. E., Gallagher, C. G., and Govan, J. R. W. (2002). A randomised clinical trial of nebulised tobramycin or colistin in cystic fibrosis. Eur. Respir. J. 20 (3), 658-664. doi: 10.1183/09031936.02.00248102

Kerr, J. (1994). Inhibition of fungal growth by Pseudomonas aeruginosa and Pseudomonas cepacia isolated from patients with cystic fibrosis. J. Infect. 28 (3), 305-310. doi: 10.1016/S0163-4453(94)91943-7

Khan, M. A., Ali, Z. S., Sweezey, N., Grasemann, H., and Palaniyar, N. (2019). Progression of Cystic Fibrosis Lung Disease from Childhood to Adulthood: Neutrophils, Neutrophil Extracellular Trap (NET) Formation, and NET Degradation. Genes 10 (3), 183. doi: 10.3390/genes10030183

Kimura, S., Odawara, J., Aoki, T., Yamakura, M., Takeuchi, M., and Matsue, K. (2009). Detection of sputum Aspergillus galactomannan for diagnosis of invasive pulmonary aspergillosis in haematological patients. Int. J. Hematol. 90 (4), 463-470. doi: 10.1007/s12185-009-0429-8

Kitasato, Y., Tao, Y., Hoshino, T., Tachibana, K., Inoshima, N., Yoshida, M., et al. (2009). Comparison of Aspergillus galactomannan antigen testing with a new cut-off index and Aspergillus precipitating antibody testing for the diagnosis of chronic pulmonary aspergillosis. Respirology (Carlton Vic.) 14 (5), 701-708. doi: $10.1111 / j .1440-1843.2009 .01548 . x$
Kraemer, R., Deloséa, N., Ballinari, P., Gallati, S., and Crameri, R. (2006). Effect of Allergic Bronchopulmonary Aspergillosis on Lung Function in Children with Cystic Fibrosis. Am. J. Respir. Crit. Care Med. 174 (11), 1211-1220. doi: 10.1164/rccm.200603-423OC

Lammertyn, E. J., Vandermeulen, E., Bellon, H., Everaerts, S., Verleden, S. E., Van Den Eynde, K., et al. (2017). End-stage cystic fibrosis lung disease is characterised by a diverse inflammatory pattern: an immunohistochemical analysis. Respir. Res. 18 (1), 10. doi: 10.1186/s12931-016-0489-2

Liu, J. C., Modha, D. E., and Gaillard, E. A. (2013). What is the clinical significance of filamentous fungi positive sputum cultures in patients with cystic fibrosis? J. cystic fibrosis Off. J. Eur. Cystic Fibrosis Soc. 12 (3), 187. doi: 10.1016/ j.jcf.2013.02.003

Lum, S., Gustafsson, P., Ljungberg, H., Hulskamp, G., Bush, A., Carr, S. B., et al. (2007). Early detection of cystic fibrosis lung disease: multiple-breath washout versus raised volume tests. Thorax 62 (4), 341-347. doi: 10.1136/ thx.2006.068262

McMahon, M. A., Chotirmall, S. H., McCullagh, B., Branagan, P., McElvaney, N. G., and Logan, P. M. (2012). Radiological abnormalities associated with Aspergillus colonization in a cystic fibrosis population. Eur. J. Radiol. 81 (3), e197-e202. doi: 10.1016/j.jrad.2011.01.114

Mennink-Kersten, M. A., Donnelly, J. P., and Verweij, P. E. (2004). Detection of circulating galactomannan for the diagnosis and management of invasive aspergillosis. Lancet Infect. Dis. 4 (6), 349-357. doi: 10.1016/S1473-3099(04) 01045-X

Miller, M. R., Hankinson, J., Brusasco, V., Burgos, F., Casaburi, R., Coates, A., et al. (2005). Standardisation of spirometry. Eur. Respir. J. 26 (2), 319-338. doi: 10.1183/09031936.05.00034805

Nagano, Y., Elborn, J. S., and Millar, B. C. (2010). Comparison of techniques to examine the diversity of fungi in adult patients with cystic fibrosis. Med. Mycol. 48 (1), 166-176.el. doi: 10.3109/13693780903127506

Nguyen, M. H., Jaber, R., Leather, H. L., Wingard, J. R., Staley, B., Wheat, L. J., et al. (2007). Use of Bronchoalveolar Lavage To Detect Galactomannan for Diagnosis of Pulmonary Aspergillosis among Nonimmunocompromised Hosts. J. Clin. Microbiol. 45 (9), 2787-2792. doi: 10.1128/JCM.00716-07

Noni, M., Katelari, A., Dimopoulos, G., Doudounakis, S., Tzoumaka-Bakoula, C., and Spoulou, V. (2015). Aspergillus fumigatus chronic colonization and lung function decline in cystic fibrosis may have a two-way relationship. Eur. J. Clin. Microbiol. Infect. Dis. 34 (11), 2235-2241. doi: 10.1007/s10096-015-2474-y

Nuttall, A. G. L., Velásquez, W., Beardsmore, C. S., and Gaillard, E. A. (2019). Lung clearance index: assessment and utility in children with asthma. Eur. Respir. Rev. 28 (154), 190046. doi: 10.1183/16000617.0046-2019

Oever, J. T., and Netea, M. G. (2014). The bacteriome-mycobiome interaction and antifungal host defense. Eur. J. Immunol. 44 (11), 3182-3191. doi: 10.1002/ eji.201344405

Ordonez, C. L., Henig, N. R., Mayer-Hamblett, N., Accurso, F. J., Burns, J. L., Chmiel, J. F., et al. (2003). Inflammatory and Microbiologic Markers in Induced Sputum after Intravenous Antibiotics in Cystic Fibrosis. Am. J. Respir. Crit. Care Med. 168 (12), 1471-1475. doi: 10.1164/rccm.200306-731OC

Paggiaro, P. L., Chanez, P., Holz, O., Ind, P. W., Djukanović, R., Maestrelli, P., et al. (2002). Sputum induction. Eur. Respir. J. 20 (37), 3s-8s. doi: 10.1183/ 09031936.02 .00000302

Parker, D. (2018). SOP: Cytoslide Analysis Sputum Differential Cell (Respiratory Biomedical Research Unit).

Pashley, C. H., Fairs, A., Morley, J. P., Tailor, S., Agbetile, J., Bafadhel, M., et al. (2012). Routine processing procedures for isolating filamentous fungi from respiratory sputum samples may underestimate fungal prevalence. Med. Mycology 50 (4), 433-438. doi: 10.3109/13693786.2011.615762

Peetermans, M., Goeminne, P., De Boeck, C., and Dupont, L. J. (2014). IgE Sensitization to Aspergillus fumigatus Is Not a Bystander Phenomenon in Cystic Fibrosis Lung Disease. Chest 146 (3), e99-e100. doi: 10.1378/chest.140635

Pihet, M., Carrere, J., Cimon, B., Chabasse, D., Delhaes, L., Symoens, F., et al. (2009). Occurrence and relevance of filamentous fungi in respiratory secretions of patients with cystic fibrosis - a review. Med. Mycology 47 (4), 387-397. doi: $10.1080 / 13693780802609604$

Public Health England. (2019). "Investigation of bronchoalveolar lavage, sputum and associated specimens." UK Standards for Microbiology Investigations. B 57 Issue 3.5. 
Ramsey, K. A., Ranganathan, S., Park, J., Skoric, B., Adams, A., Simpson, S. J., et al. (2014). Early Respiratory Infection Is Associated with Reduced Spirometry in Children with Cystic Fibrosis. Am. J. Respir. Crit. Care Med. 190 (10), 11111116. doi: 10.1164/rccm.201407-1277OC

Rocchi, S., Richaud-Thiriez, B., Barrera, C., Grenouillet, F., Dalphin, J., Millon, L., et al. (2015). Evaluation of mold exposure in cystic fibrosis patients' dwellings and allergic bronchopulmonary risk. J. Cystic Fibrosis 14 (2), 242-247. doi: 10.1016/j.jcf.2015.01.003

Sagel, S. D. (2009). Impact of Pseudomonas and Staphylococcus infection on inflammation and clinical status in young children with cystic fibrosis. J. Pediatr. 154 (2), 183-188.e3. doi: 10.1016/j.jpeds.2008.08.001

Saunders, R. V., Modha, D. E., Claydon, A., and Gaillard, E. A. (2016). Chronic Aspergillus fumigatus colonization of the pediatric cystic fibrosis airway is common and may be associated with a more rapid decline in lung function. Med. Mycology 54 (5), 537-543. doi: 10.1093/mmy/myv119

Sly, P. D., Brennan, S., Gangell, C., de Klerk, N., Murray, C., Mott, L., et al. (2009). Lung Disease at Diagnosis in Infants with Cystic Fibrosis Detected by Newborn Screening. Am. J. Respir. Crit. Care Med. 180 (2), 146-152. doi: 10.1164/ rccm.200901-00690C

Strieter, R. M. (2002). Interleukin-8: a very important chemokine of the human airway epithelium. Am. J. Physiol. Lung Cell. Mol. Physiol. 283 (4), L688-L689. doi: 10.1152/ajplung.00146.2002

Sudfeld, C. R., Dasenbrook, E. C., Merz, W. G., Carroll, K. C., and Boyle, M. P. (2009). Prevalence and risk factors for recovery of filamentous fungi in individuals with cystic fibrosis. J. Cystic Fibrosis 9 (2), 110-116. doi: 10.1016/ j.jcf.2009.11.010

Talento, A. F., Dunne, K., Joyce, E. A., Palmer, M., MSc, M. B. A., Johnson, E., et al. (2017). A prospective study of fungal biomarkers to improve management of invasive fungal diseases in a mixed specialty critical care unit. J. Crit. Care 40, 119-127. doi: 10.1016/j.jcrc.2017.03.025

Thia, L. P., and Balfour Lynn, I. M. (2009). Diagnosing allergic bronchopulmonary aspergillosis in children with cystic fibrosis. Paediatric Respir. Rev. 10 (1), 3742. doi: 10.1016/j.prrv.2009.01.001
Tracy, M. C., and Moss, R. B. (2018). The myriad challenges of respiratory fungal infection in cystic fibrosis. Pediatr. Pulmonol. 53 (S3), S75-S85. doi: 10.1002/ ppul.24126

Walicka-Serzysko, K., Postek, M., Milczewska, J., and Sands, D. (2019). Change in lung clearance index with microbiological status in children with cystic fibrosis. Pediatr. Pulmonol. 54 (6), 729-736. doi: 10.1002/ppul.24278

Conflict of Interest: DP reports grants from Gilead Sciences Grant Program, outside the submitted work. This grant was awarded for a separate ongoing study termed: Changes in the Cystic Fibrosis Airway Mycobiome Over Time and Its Link with Bacterial Species Rarefication. CP has received research support and honorariums for advisory board from Pulmocide who are developing a new antifungal agent. EG reports from Consultancy work for Boehringer Ingelheim November 2016, from Consultancy work for Anaxsys July 2018, grants from Circassia research grant, grants from Gilead sciences research grant, grants from Chiesi Limited, from Medimmune research collaboration, and outside the submitted work: 1. Consultancy work for Boehringer Ingelheim November 2016. Money, paid to the institution (The University of Leicester); 2. Consultancy work for Anaxsys July 2018, money paid to the institution (The University of Leicester); 3. Circassia research grant, investigator-led research grant; 4. Gilead sciences research grant, investigator-led research grant; and 5. Medimmune research collaboration, research collaboration.

The remaining author declares that the research was conducted in the absence of any commercial or financial relationships that could be construed as a potential conflict of interest.

Copyright (C) 2021 Patel, Dacanay, Pashley and Gaillard. This is an open-access article distributed under the terms of the Creative Commons Attribution License (CC BY). The use, distribution or reproduction in other forums is permitted, provided the original author(s) and the copyright owner(s) are credited and that the original publication in this journal is cited, in accordance with accepted academic practice. No use, distribution or reproduction is permitted which does not comply with these terms. 